\title{
Os idosos e as barreiras de acesso às novas tecnologias da informação e comunicação
}

\author{
Marília Matias Kestering Tavares - Universidade Federal de Santa Catarina \\ marilia.kestering@gmail.com
}

\section{Samara Tomé Correa de Souza - Universidade Federal de Santa Catarina samaratc@gmail.com}

\begin{abstract}
Resumo: As barreiras que dificultam a inclusão digital dos idosos são diversas. Algumas das dificuldades encontradas referem-se aos declínios sensoriais, motores, físicos decorrentes do avanço da idade. Sendo que a difusão de novas tecnologias tem exigido dos idosos um aprendizado contínuo, para que os mesmos possam interagir de forma autônoma com os aparatos tecnológicos. Este artigo apresenta algumas questões relevantes à inclusão digital relacionada à terceira idade. Propõe-se observar a necessidade de desenvolver e oportunizar formas alternativas de acesso às novas tecnologias para o público idoso. Discutem-se as possíveis limitações que a terceira idade encontra para interagir com as novas tecnologias. É abordado o direito a inclusão digital para pessoas idosas e de que forma isto é definido na legislação vigente. Para podermos compreender quais peculiaridades devem ser observadas ao desenvolver novas tecnologias que favoreçam a integração das pessoas idosas com os avanços tecnológicos.
\end{abstract}

Palavras-chaves: Inclusão Digital, Educação ao Longo da Vida, Tecnologias da Informação e Comunicação.

\section{The elderly and barriers to access new information and communication technologies}

\begin{abstract}
There is a plenty of barriers which difficult to digital inclusion of the older people. The declines in sensory, motors and physical arising from aging and the diffusion of new technologies requiring a continuous learning of the users are some of the difficulties found. This article presents some issues relevant to digital inclusion related to seniors. It is proposed to observe the need to develop alternative ways to create access to new technologies for the elderly public. Discuss the possible limitations of the elderly to interact with the new technologies. It address the entitlement digital insertion for the elderly and how it is defended by law. In order to understand the peculiarities which must be met by developing new technologies that promote the integration of older people with technological advances.
\end{abstract}

Keywords: Digital Inclusion, Lifelong Education, Information Technology and Communication.

\section{Introdução}

O crescimento da população idosa tem se configurado como uma tendência mundial. Os avanços da medicina, da farmacologia, das melhorias sanitárias (higiene e saúde) e do reconhecimento dos direitos dos idosos podem ser considerados alguns dos fatores que colaboram para a melhoria da qualidade de vida, e consequentemente para um aumento da expectativa de vida e crescimento da população idosa. 
Os direitos de toda população brasileira são reconhecidos pela Constituição Federal de 1988. A terceira idade, além da Constituição, é amparada pelo Estatuto do Idoso, sancionado no ano de 2003. Este lhes confere direitos diferenciados, reconhecendo que o idoso possui características e necessidades diferentes do restante da população. Um dos pontos relevantes do Estatuto do Idoso é a preocupação com a interação do idoso com a vida moderna, com ênfase na necessidade de interação com os aparatos tecnológicos, para que este público tenha mais autonomia ao executar tarefas cotidianas, como por exemplo: usar um caixa eletrônico sem a necessidade de ser assessorado por um atendente.

Segundo Czaja e Lee (2007), "não ter acesso e ser capaz de usar a tecnologia cada vez mais colocará os idosos em desvantagem em termos de sua capacidade de viver e funcionar independentemente". As novas tecnologias da informação e comunicação (NTIC) podem ser consideradas um dos fatores que mais segregam a população idosa na atualidade. É comum que parte da população idosa apresente no decorrer de sua vida dificuldades de ordem cognitiva, motora, talvez financeira, que podem ser fatores limitantes ao acesso às novas tecnologias.

Este artigo tem por objetivo discutir questões referentes ao tema informática para a terceira idade, observando a necessidade de desenvolver e oportunizar formas alternativas de acesso as NTIC. Nas próximas seções serão abordados temas como a população idosa no Brasil, será apresentada uma breve análise da população idosa. $\mathrm{O}$ idoso e o direito de acesso às NTIC, uma contextualização da legislação vigente. Os declínios decorrentes da idade e suas implicações na interação com as NTIC, observamse as possíveis limitações que os idosos podem apresentar. A acessibilidade como alternativa para facilitar a interação humano computador. A tecnologia assistiva e sua aplicação para a terceira idade, esta apresentada como uma forma de superar as limitações apresentadas pelos idosos, e por fim, algumas considerações.

\section{A população idosa no Brasil}

Os censos populacionais entre 2000 e 2010, mostram um índice de crescimento da população brasileira de $12,3 \%$, aproximadamente 21 milhões de pessoas, no total contabilizado em 2010 de aproximadamente 191 milhões de habitantes. A proporção de idosos na população neste mesmo período passou de $5,9 \%$ para $7,4 \%$, aproximadamente 20 milhões de pessoas (IBGE, 2012). Na figura 1 podemos observar a estimativa de crescimento da população por sexo, a partir dos 60 anos e como esta tende a crescer.
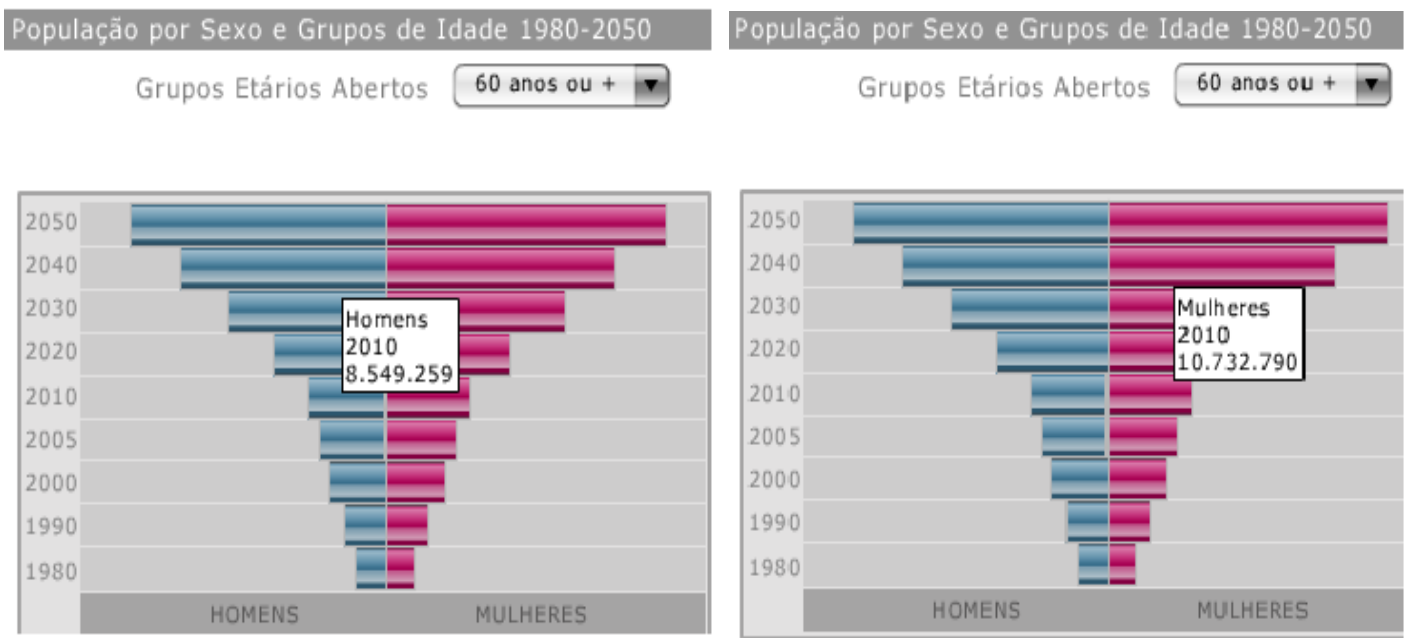

Figura 1 - População por Sexo e Grupos de Idade 1980-2050. 
Este aumento da população idosa tende a modificar o formato da pirâmide populacional. $\mathrm{O}$ aumento da expectativa de vida e o declínio da taxa de fecundidade, fez com que se iniciasse um processo contínuo de estreitamento da base da pirâmide etária, consequentemente, de envelhecimento da população (Garcia \& Carvalho, 2003). Provocando o que os pesquisadores chamam de processo de retangularização da pirâmide populacional.

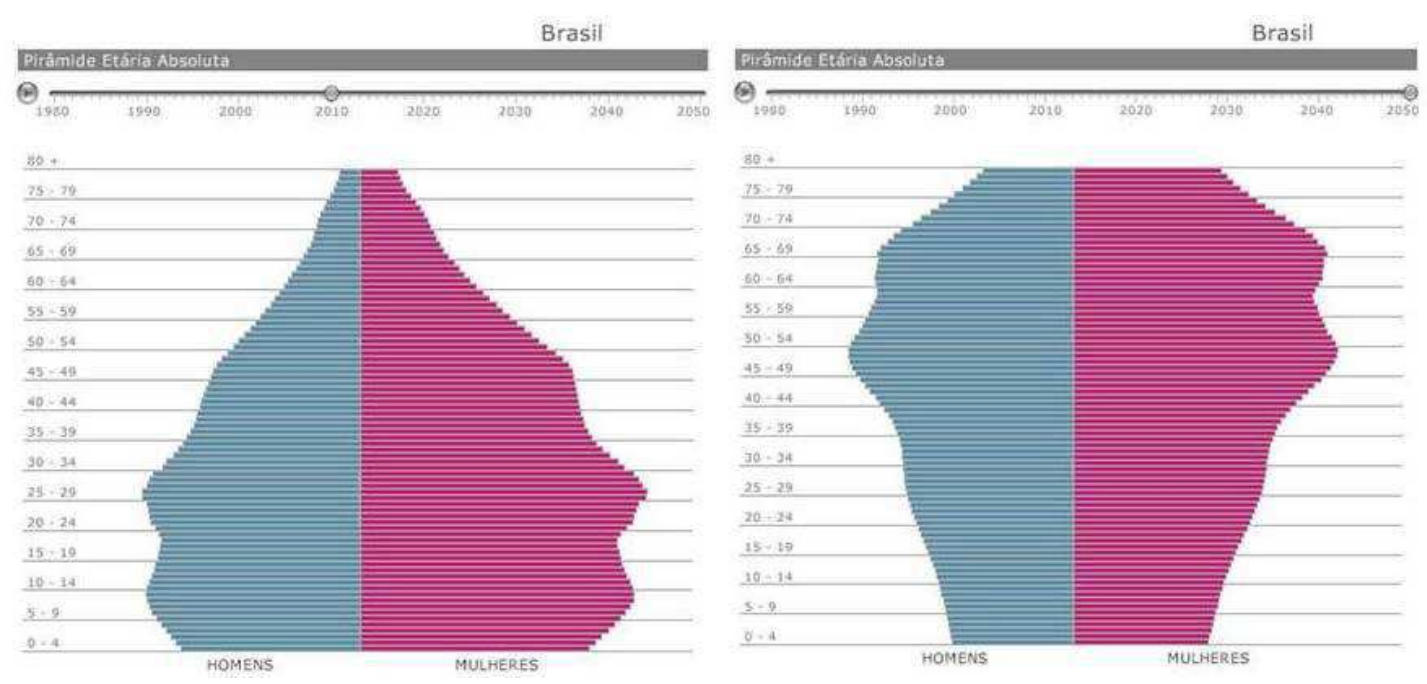

Figura 2 - Pirâmide etária absoluta.

Conforme a figura 2 é notável a mudança no perfil da população brasileira nos próximos anos. Assim, é necessário que os órgãos governamentais criem políticas públicas de saúde, educação, cultura, lazer, inclusão digital para que as necessidades desses idosos sejam atendidas e para que eles não se sintam isolados da sociedade.

\section{Os Idosos e o direito de acesso as novas tecnologias}

A Constituição Federal de 1988 que dispõe sobre os direitos dos cidadãos brasileiros estabelece no capítulo III, seção I, Art. 205 que a educação é um direito de todos e dever do Estado e da família, será promovida e incentivada com a colaboração da sociedade, visando ao pleno desenvolvimento da pessoa, seu preparo para o exercício da cidadania e sua qualificação para o trabalho (BRASIL, 1988). Este direito é garantido a todo brasileiro, sem distinção de raça, cor ou idade. O Estatuto do Idoso, no Art. 3 prevê a viabilização de formas alternativas de participação, ocupação e convívio do idoso com as demais gerações. Assim como no Art. $21, \S 1^{\circ}$ garante que os cursos especiais para idosos incluirão conteúdo relativo às técnicas de comunicação, computação e demais avanços tecnológicos, para sua integração à vida moderna (BRASIL, 2003).

Ao longo do tempo o conceito de cidadania foi se construindo e modificando, por exemplo, na Grécia antiga apenas uma parte da população podia usufruir dessa condição, ou mesmo na Revolução Francesa, em que a cidadania era construída de forma hierárquica. Porém na sociedade contemporânea a cidadania fundamenta-se na ideia de que cada pessoa é um sujeito de direitos. No que se refere às pessoas com deficiência, isto significa que o indivíduo não deve ser mais visto como alguém dependente de cuidados ou que precisa permanentemente de assistência, mas como uma pessoa com voz e vontade próprias. Ser sujeito de direitos significa que qualquer um, ou qualquer uma, tem o direito de ter direitos (UNESCO, 2007). 
Ao abordar este texto apresentado pela UNESCO não se tem a intenção de classificar o idoso como um deficiente, mas sim propor a discussão de que tanto o idoso quanto a pessoa com deficiência, deve requerer e exercer seus direitos, para garantir que sua voz e vontade sejam ouvidas. Sendo assim, é dever do Estado garantir aos jovens, deficientes e ao adulto idoso o acesso à educação. No que diz respeito ao adulto idoso, o Estado deve oferecer recursos não apenas para educação escolar regular, mais também para o ensino das novas tecnologias (inclusão digital). Para que os idosos consigam exercer a sua cidadania, sem o sentimento de exclusão da sociedade por não acompanhar os avanços tecnológicos.

Promover ações e políticas de inclusão digital é uma forma de oportunizar ao idoso a possibilidade de usufruir da gama de conhecimento que na atualidade estão disponíveis nos ambiente web (revistas, jornais, portais acadêmicos, blogs).

\section{Os declínios decorrentes da idade e suas implicações na interação com as NTIC}

Faz parte do nosso ciclo de vida o envelhecimento. O fato de envelhecermos traz consigo uma série de declínios de ordem fisiológica, sensorial, cognitiva, emocional etc. (SALES, 2002). Nos últimos anos a qualidade de vida dos idosos melhorou significativamente se comparada com as gerações anteriores. A incorporação de novos hábitos (mais saudáveis), o acompanhamento médico e fatores sociais têm contribuído para o aumento da expectativa de vida da população idosa. Porém mesmo com os avanços da medicina é possível que os idosos apresentem indícios de declínios de saúde que são próprios do processo de envelhecimento.

Czaja e Lee (2007) afirma que a probabilidade de uma pessoa desenvolver uma doença crônica ou cognitiva aumenta com a idade, e muitos idosos possuem ao menos uma condição crônica. Segundo Sales (2007) é comum à medida que a pessoa envelhece ocorrer alterações como: redução da capacidade de memória de curto termo, acuidade visual, audição, motricidade fina, locomoção e outras. Estes fatores podem constituir-se como barreira de acesso dos idosos com as NTIC.

Os declínios decorrentes da idade têm implicações no design de interfaces e sistemas técnicos. Por exemplo, embora a maioria dos idosos não experimente deficiências visuais severas, muitos experimentam declínios na visão suficientes para tornar difícil a percepção e compreensão de informações visuais (CZAJA, LEE, 2007). Para minimizar os impactos destes declínios no acesso as NTIC, seria importante desenvolver interfaces mais acessíveis aos idosos, respeitando algumas critérios de ergonomia e usabilidade.

\section{Interface Humano-Computador: interação com o idoso}

$\mathrm{O}$ avanço da tecnologia proporcionou às pessoas do mundo todo comunicar-se entre si com muita facilidade, de maneira especial através da web. Segundo Nielsen (2000) no início a web era acessada apenas por pessoas que detinham o conhecimento da tecnologia avançada. Mas com a crescente popularização da web, houve uma diversificação dos usuários. O que evidencia a necessidade de pensar em diretrizes de acessibilidade na web para esses usuários diversificados.

Gregor et al. (2002) cita que as interfaces humano-computador para a maior parte dos sistemas computacionais de uso geral foram desenhadas para um usuário "típico" e jovem. Dessa forma grande parte do público idoso encontra dificuldades de interação com as interfaces desenvolvidas atualmente. A web poderia ser ainda mais democrática caso seus desenvolvedores preocupassem com a diversidade de usuários 
existentes. Mas, infelizmente o que se pode observar é que a maioria dos sites não seguem quaisquer diretrizes de acessibilidade propostas para web. $\mathrm{O}$ que dificulta a interação humano-computador.

Para Godinho (2010) "acessibilidade consiste na facilidade de acesso e de uso de ambientes, produtos e serviços por qualquer pessoa e em diferentes contextos". Neste sentido, a inclusão digital do idoso requer que os componentes de entrada e saída de dados, as interfaces sejam desenvolvidas dentro de padrões de acessibilidade, que não ofereçam obstáculos para o usuário idoso, ou que as minimizem. As equipes de desenvolvimento web, de software ou hardware poderiam ser mais sensíveis às especificidades deste público, pois facilitar para o idoso não é dificultar para os mais jovens.

Sabe-se que existe a necessidade de criar equipamentos e materiais inclusivos (para idosos e outras minorias), adequados a cada especificidade e não fazer que o público se adapte a aos produtos desenvolvidos. A partir desses pressupostos e com essas preocupações, podemos pensar em formas alternativas de permitir a inclusão digital dos idosos que apresentam algum declínio funcional, decorrente do processo de envelhecimento. Neste sentido, a tecnologia assistiva apresenta-se como uma alternativa para promover a interação humano-computador.

\section{Tecnologia Assistiva e sua aplicação para a terceira idade}

A tecnologia assistiva (TA) pode ser compreendida como toda ferramenta, recurso ou estratégia e processo desenvolvido e utilizado com a finalidade de proporcionar maior independência e autonomia à pessoa com deficiência (UNESCO, 2007). A TA deve então ser entendida como um auxílio que promoverá a ampliação de uma habilidade funcional deficitária ou possibilitará a realização da função desejada e que se encontra impedida por circunstância de deficiência ou pelo envelhecimento (BERSCH, 2008).

No que se referem às NTIC, estas podem ser utilizadas como ou por meio de TA. São utilizadas como TA, quando a ajuda técnica para se alcançar determinado objetivo é provida pelo uso do computador, a citar, o uso do computador como caderno eletrônico para o indivíduo que não consegue escrever no caderno de papel. Porém, utilizam-se as NTIC por meio da TA, quando se almeja utilizar o próprio computador, necessitando dessa forma de algumas ajudas técnicas especificas que permitam ou facilitem tal tarefa. Por exemplo, adaptações de teclado, de mouse, software especiais. Neste sentido, no ambiente computacional a TA pode ser utilizada com a finalidade de permitir a interação humano-computador por pessoas com diferentes graus de comprometimento motor, sensorial e ou de linguagem (UNESCO, 2007).

Para Bersch (2008) "o objetivo maior da TA é proporcionar à pessoa com deficiência maior independência, qualidade de vida e inclusão social, através da ampliação de sua comunicação, mobilidade, controle de seu ambiente, habilidades de seu aprendizado e trabalho". Com base na TA é possível desenvolver componentes de hardwares e softwares que sejam mais acessíveis para pessoas com dificuldades sensoriais ou motoras. As ferramentas que são desenvolvidas para pessoas com deficiência podem ser adaptadas para suprir os declínios de ordem motora ou sensorial que os idosos possuem ou que venham desenvolver. Pois, "a maior parte das pessoas com deficiência são idosas. A deficiência aumenta em paralelo com a longevidade das pessoas" (GODINHO, 2010). Dessa forma torna-se imprescindível investir no desenvolvimento de TA, seja para promover a inclusão social ou digital dessas pessoas. 


\section{Considerações Finais}

No cenário atual o domínio das NTIC é indispensável para a interação com a vida moderna, visto que está em todos os lugares, em casa: nos aparelhos eletrodomésticos, nos bancos: uso dos caixas eletrônicos, nos supermercados, no uso de aparelhos celulares entre muitos outros. Por isso devemos pensar em formas de incluir os idosos neste novo ambiente informatizado, para que não se sintam excluídos da sociedade. Sendo fundamental fomentar iniciativas que promovam a inclusão digital deste público, reconhecendo sua cidadania e capacidade de ter uma vida ativa.

Sabendo que a população idosa tende a aumentar significativamente segundo projeções do IBGE, e que estas pessoas tem a probabilidade de desenvolver alguma doença crônica ao longo da vida, é preciso desenvolver e oportunizarmos formas alternativas de acesso às novas tecnologias para este público. A tecnologia assistiva surge com esta característica, de respeitar as limitações que por ventura, o idoso venha desenvolver e oferecer uma possível solução aos problemas encontrados pela terceira idade para interagir com as NTIC. Em suma, ao desenvolvermos interfaces apropriadas para os idosos, estamos de certa forma desenvolvendo algo que poderemos usufruir no futuro, pois os jovens de hoje são os idosos de amanhã.

\section{Agradecimentos}

À Universidade Federal de Santa Catarina por nos conceder a oportunidade de realizar este trabalho e em especial a professora Márcia Barros de Sales.

\section{Referências}

BERSCH, Rita. Introdução à tecnologia assistiva. Porto Alegre: CEDI, 2008. Disponível em: <http://www.assistiva.com.br/Introducao\%20TA\%20Rita\%20Bersch.pdf>. Acesso em $30 / 04 / 2012$.

BRASIL. Constituição. (1988). Constituição da República Federativa do Brasil: promulgada em 5 de outubro de 1988. Disponível em:<http://www.planalto.gov.br/ccivil_03/constituicao/constitui\%C3\%A7ao.htm>.

Acesso em: 30/04/2012.

BRASIL. Estatuto do Idoso. (2003). Disponível em: <http://www.planalto.gov.br/ccivil/leis/2003/L10.741.htm> . Acesso em: 30/04/2012.

CARVALHO, José Alberto Magno de; GARCIA, Ricardo Alexandrino. O envelhecimento da população brasileira: um enfoque demográfico. Cad. Saúde Pública [online]. 2003, vol.19, n.3, pp. 725-733.

CJAZA, Sara J.; LEE, Chin Chin. The impact of aging on access to technology. In Universal Access in the Information Society, 2007.

GREGOR, Peter, NEWELL, Alan F., ZAJICEK, Mary. Designing for Dynamic Diversity - interfaces for older people. Proceedings of the Fifth International ACM SIGCAPH Conference on Assistive Technologies Edinburgh, July 2002. 
GODINHO, Francisco Alexandre Ferreira Biscaia. Uma nova abordagem para a formação de engenharia de reabilitação em Portugal. Vila Real, 2010. Tese (Doutorado) - Universidade de Trás-os-Montes e Alto Douro, Engenharia Electrotécnica e de Computadores.

IBGE. Projeção da População: Brasil. Disponível em <http://www.ibge.gov.br/home/estatistica/populacao/projecao_da_populacao/2008/pira mide/piramide.shtm>. Acesso em 10/05/2012.

SALES, Márcia Barros de. Desenvolvimento de um checklist para a avaliação de acessibilidade da web para usuários idosos. Florianópolis, 2002. 121 f. Dissertação (Mestrado) - Universidade Federal de Santa Catarina, Centro Tecnológico. Programa de Pós-Graduação em Engenharia de Produção.

SALES, Márcia Barros de. Modelo multiplicador utilizando a aprendizagem por pares focado no idoso. Florianópolis, 2007. 138 f. Tese (Doutorado) - Universidade Federal de Santa Catarina, Centro Tecnológico. Programa de Pós-graduação em Engenharia e Gestão do Conhecimento.

UNESCO. Inclusão digital e social de pessoas com deficiências: textos de referência para monitores de telecentros.- Brasília, 2007. 73p. 